OPEN ACCESS

Edited by:

Simon Rousseau,

McGill University, Canada

Reviewed by:

Li-Wha Wu,

National Cheng Kung University,

Taiwan

Wei-Hsiung Yang,

Mercer University, United States

*Correspondence:

Dingli Xu

dinglixu@fimmu.com;

dlxugz@163.com

Qingchun Zeng

qingchunzeng@smu.edu.cn;

z-qch@163.com

${ }^{\dagger}$ These authors have contributed equally to this work

Specialty section: This article was submitted to Signaling,

a section of the journal Frontiers in Cell and Developmental Biology

Received: 16 September 2019 Accepted: 22 January 2020

Published: 14 February 2020

Citation:

Zhou P, Li Q, SU S, Dong W

Zong S, Ma $Q$, Yang $X$, Zuo D, Zheng S, Meng $X, X u D$ and Zeng $Q$ (2020) Interleukin 37 Suppresses M1

Macrophage Polarization Through Inhibition of the Notch1 and Nuclear

Factor Kappa B Pathways.

Front. Cell Dev. Biol. 8:56.

doi: 10.3389/fcell.2020.00056

\section{Interleukin 37 Suppresses M1 Macrophage Polarization Through Inhibition of the Notch1 and Nuclear Factor Kappa B Pathways}

\author{
Peitao Zhou't, Qianqin $\mathrm{Li}^{2+}$, Shuwen Su${ }^{1+}$, Wenhui Dong ${ }^{1}$, Suyu Zong ${ }^{1}$, Qiong Ma', \\ Xi Yang ${ }^{1}$, Daming Zuo ${ }^{3}$, Shaoyi Zheng ${ }^{2}$, Xianzhong Meng ${ }^{4}$, Dingli $X^{1,5 *}$ and \\ Qingchun Zeng ${ }^{1,4,5 *}$
}

${ }^{1}$ Key Laboratory for Organ Failure Research, Department of Cardiology, Nanfang Hospital, Southern Medical University, Guangzhou, China, ${ }^{2}$ Department of Cardiovascular Surgery, Nanfang Hospital, Southern Medical University, Guangzhou, China, ${ }^{3}$ Department of Immunology, School of Basic Medical Sciences, Southern Medical University, Guangzhou, China, ${ }^{4}$ Department of Surgery, University of Colorado Denver, Aurora, CO, United States, ${ }^{5}$ Guangzhou Regenerative Medicine and Health Guangdong Laboratory, Guangzhou, China

Macrophage-orchestrated chronic inflammation plays an important role in cardiovascular disease, including accelerating the development of calcific aortic valve disease (CAVD). M1 and M2 macrophage polarization imbalances can alter intensity of inflammatory responses. Recombinant human interleukin 37 (IL-37) could be involved in regulating immune cell function to attenuate inflammation. This study aimed to identify IL-37 specifically modulates M1 polarization and investigate the underlying mechanism. Compared with normal valves, there are more M1 macrophages accumulation and less IL-37 expression in calcific aortic valves, which may indicate a negative relationship between IL-37 and M1 polarization. THP-1 cells could differentiate into resting macrophages with phorbol-12-myristate-13-acetate (PMA) and then polarize into M1 macrophages following treatment with lipopolysaccharide (LPS) and interferon gamma (IFN- $\gamma)$. In vitro, recombinant human IL-37 attenuated the expression of inducible nitric oxide synthase (iNOS), CD11C, IL-6 and monocyte chemoattractant protein 1 (MCP-1) in M1 but augmented the expression of CD206 and IL-10 in M2. The suppression of $\mathrm{M} 1$ polarization was associated with the inhibition of the activation of the nuclear factor kappa B (NF-кB) and Notch1 signaling pathways. These results demonstrated that IL-37 inhibits the macrophages polarizing into M1 type via the inhibition of the Notch1 and nuclear factor kappa B pathways. In summary, IL-37 could be a potential therapeutic candidate for progressive CAVD by modulating M1 polarization and its orchestrated inflammation.

Keywords: interleukin 37, macrophage polarization, Notch1, NF-кB, calcific aortic valve disease

\section{INTRODUCTION}

Calcific aortic valve disease (CAVD) is recognized as a chronic progressive inflammatory disease and has become a leading cause of cardiovascular disease in people aged 65 years or older and the second most common indication for cardiac surgery (Mohler et al., 2001; Rajamannan et al., 2011). However, the mechanism underlying CAVD development remains incompletely understood. 
But examinations of calcific human aortic valves indicate that macrophages may play an important role in the calcification process (Hjortnaes et al., 2010).

Macrophages are myeloid immune cells that coordinate inflammatory processes. Macrophages could assume two major functional phenotypes: the classical activation phenotype, also called M1 macrophages, and the alternative activation phenotype, also known as M2 macrophages (Liu et al., 2014). M1 macrophages can be induced by Toll-like receptor (TLR) ligands and IFN- $\gamma$ while M2 by IL4/IL13 (M2a), immune complex (M2b), and the anti-inflammatory cytokines IL-10 or transforming growth factor- $\beta$ (M2c) (Zhou et al., 2014). M1 macrophages express numerous pro-inflammatory cytokines, such as IL-1 $\beta$, IL-6, IL-23, inducible nitric oxide synthase (iNOS) and tumor necrosis factor alpha (TNF- $\alpha$ ), while M2 macrophages secrete molecules including Arginase1 (Arg1), IL-10 and transforming growth factor- $\beta 1$ (TGF- $\beta 1$ ) which are associated with parasite infestation, tissue remodeling and tumor progression (Sica and Mantovani, 2012; Liu et al., 2014). Notably, studies have shown that M1 and M2 are found in the central portions of atherosclerotic and calcific lesions and convert into each other in response to micro-environmental changes (Mantovani et al., 2013; Liu et al., 2014). The finding that M1 macrophages accumulate in aortic valvular lesions suggests that the inflammation induced by M1 macrophages is pivotal in cardiovascular calcification (Li et al., 2016).

However, the mechanism underlying macrophage polarization is complicated and may involve various signaling pathways that affect one another. Many signaling pathways, such as Akt2 (Liu et al., 2019), activator protein 1 (AP-1) (Liu et al., 2014), interferon-regulatory factor (IRF) (Satoh et al., 2010), peroxisome proliferator-activated receptor gamma (PPAR$\gamma$ ) (Odegaard et al., 2007), hypoxia-inducible factor -2alpha

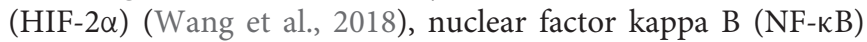
(Oeckinghaus et al., 2011) and Notch1 signaling pathways (Wang Y. et al., 2010), are involved in macrophage polarization. The stimulation by lipopolysaccharide (LPS) plus interferon gamma (IFN- $\gamma$ ) induces the polarization of resting macrophages into activated M1 macrophages (Foldi et al., 2016).

IL-37, also known as IL-1F7, a member of the IL-1 family, has emerged as a potential suppressor of inflammation (Dinarello and Bufler, 2013). The protein has been reported in human monocytes, macrophages, epithelial cells, aortic valve interstitial cells, tonsil plasma cells and breast carcinoma cells, but has not been detected in murine (Nold et al., 2010). IL-37 has been observed to exert anti-inflammatory effects on the innate and acquired immune responses both in vitro and in vivo (Boraschi et al., 2011). Recombinant human IL-37 attenuates pro-inflammatory cytokine production in mouse RAW cells, monocytes and epithelial cells and alleviates inflammation-induced injury in wild-type mice (Nold et al., 2010; Dinarello et al., 2016). Notably, our groups have proven that IL-37 transgenic mice are protected from the aortic valve lesions induced by inflammation (Zeng et al., 2017). However, whether IL-37 suppresses macrophage polarization to inhibit inflammation has not yet been clearly determined.
In this study, we aimed to determine whether IL-37 suppresses M1 polarization to inhibit inflammation and to explore the mechanism by which IL-37 exerts its effect. We examined the expression of the M1/M2 macrophage phenotypes in calcific and non-calcified aortic valves, evaluated the effect of recombinant IL-37 on macrophage polarization and investigated whether IL-37 modulates M1 macrophage polarization via the NF- $\kappa$ B and Notch1 pathways.

\section{MATERIALS AND METHODS}

\section{Cell Culture and Treatment}

THP-1 cells were obtained from the American Type Culture Collection (ATCC). Recombinant human IL-37 (Cat. No. Ab224789) was purchased from Abcam. THP-1 cells were maintained in Roswell Park Memorial Institute (RPMI) 1640 medium (Gibco) supplemented with 10\% fetal bovine serum (FBS; Gibco) at $37^{\circ} \mathrm{C}$ in a humidified incubator with a $5 \% \mathrm{CO} 2$ atmosphere. THP-1 cells were seeded at $5 \times 10^{6}$ cells/well in 6-well plates and cultured in RPMI 1640 medium containing 10\% FBS and then were differentiated into resting (M0) macrophages by $24 \mathrm{~h}$ incubation with $100 \mathrm{ng} / \mathrm{ml}$ phorbol 12-myristate 13-acetate (PMA; Sigma-Aldrich, Cat. No. 79346) followed by $24 \mathrm{~h}$ incubation in RPMI medium (Genin et al., 2015). After culturing for $48 \mathrm{~h}$, cells were washed with phosphatebuffered saline (PBS), following by treating with or without LPS (100 ng/ml; Sigma-Aldrich, Cat. No. L2630) and IFN- $\gamma$ (20 ng/ml; Sigma-Aldrich, Cat. No. SRP3058) for $24 \mathrm{~h}$ to polarize into M1 macrophages (Genin et al., 2015; Tedesco et al., 2015). To determine the effect of IL-37 on M1 polarization, we pre-treated the M0 macrophages with IL-37 (0.1 ng/ml) (Zeng et al., 2017) $1 \mathrm{~h}$ before adding LPS and IFN- $\gamma$ to the medium (Zhou et al., 2015).

To determine the effects of NF- $\kappa \mathrm{B}$ and Notch1 on M1 polarization, we added NF- $\kappa$ B specific inhibitor BAY11-7082 (5 $\mu \mathrm{M}$; Sigma-Aldrich, Cat. No. B5556) and the $\gamma$-secretase inhibitor DAPT (50 $\mu \mathrm{M}$; Sigma-Aldrich, Cat. No. D5942) to the culture medium $1 \mathrm{~h}$ before adding LPS and IFN- $\gamma$ to the medium. To investigate the effect of Notch1 on NF- $\mathrm{B}$ phosphorylation, we added DAPT $(50 \mu \mathrm{M})$ to the culture medium $1 \mathrm{~h}$ prior to treating the cells with LPS and IFN- $\gamma$.

\section{Histology and Immunohistochemistry}

This study was approved by the Ethical Committee of Nanfang Hospital, China. Informed consent was obtained from all patients. Normal aortic valves were collected from the explanted hearts of six males (mean age $58 \pm 8.1$ years) without CAVD undergoing heart transplantation. Valves with calcification were obtained from 6 males (mean age $60 \pm 11.3$ years) undergoing aortic valve replacement.

Paraffin-embedded non-calcific and calcific aortic valve samples were cut into 5 - $\mu \mathrm{m}$-thick sections, and then were incubated for $20 \mathrm{~min}$ at $65^{\circ} \mathrm{C}$ before deparaffinization with xylene and alcohol. Hematoxylin and eosin-stained sections were examined to identify the difference between non-calcified and calcified aortic valves. For Immunohistochemistry, following antigen retrival through microwave, the prepared sections 
were incubated in $3 \% \mathrm{H}_{2} \mathrm{O}_{2}$ for $10 \mathrm{~min}$. Then the sections were rinsed with phosphate buffer saline and blocked in $5 \%$ bovine serum albumin (BSA) for $30 \mathrm{~min}$ at room temperature followed by incubation with primary antibodies against CD11c (1:200; Abcam, Cat. No. EP1347Y), CD206 (1:1000; Abcam, Cat. No. ab8918) and IL-37 (1:100; Abcam, Cat. No. ab101376) overnight at $4^{\circ} \mathrm{C}$ and horseradish peroxidaseconjugated secondary antibody for $30 \mathrm{~min}$ at room temperature. And then diaminobenzidine (DAB) was used as a chromogen to visualize positive cells. For each valve, integrated optical density (IOD)/area was counted in five representative highpowered fields in each of three slides through Image-Pro Plus 7.0. For each field, areas of interest were selected to measure IOD and area. Moreover, background IOD/area value from the directly measured IOD/area value was subtracted to acquire more accurate IOD/area value. Then, the mean value of these 15 fields was used to evaluate the target protein expression.

\section{Immunoblotting}

Briefly, total protein of aortic valve tissue samples and cell lines was extracted using protein extraction reagent (Thermo Scientific) with a cocktail of proteinase inhibitors (Fude Biological Technology) and a cocktail of phosphatase inhibitors (Fude Biological Technology) according to its protocol. The proteins were separated by $8-12 \%$ SDS-PAGE and then transferred onto PVDF membranes. After blocking with 5\% nonfat dry milk solution or $5 \%$ bovine serum albumin solution for $1 \mathrm{~h}$ at room temperature, the membranes were incubated with antibodies against iNOS (1:1000; Cell Signaling Technology, Cat. No. 2977), CD11c (1:1000; Abcam, Cat. No. EP1347Y), CD206 (1:1000; Abcam, Cat. No. ab8918), IL-37 (1:1000; Abcam, Cat. No. ab153889), IL-6 (1:1000; Abcam, Cat. No. 12153), MCP-1 (1:1000; Cell Signaling Technology, Cat. No. 39091), NICD1 (1:1000; Cell Signaling Technology, Cat. No. 3608), phosphorylated (1:1000; Cell Signaling Technology, Cat. No. 3033) and total NF-кB (1:1000; Cell Signaling Technology, Cat. No. 8242) and GAPDH (1:1000; Cell Signaling Technology, Cat. No. 5174) overnight at $4^{\circ} \mathrm{C}$ followed by incubation with horseradish peroxidase-conjugated secondary antibodies (1:5000; Fude Biological Technology) for $1 \mathrm{~h}$ at room temperature (Song et al., 2012). The protein bands were revealed using an ECL system (Thermo Fisher Scientific), and the band densities were analyzed using ImageJ software.

\section{ELISA}

Cell culture supernatants were collected, and IL-6, IL-10 and MCP-1 expression was analyzed using ELISA kits (R\&D System), according to the manufacturer's protocols.

\section{Immunofluorescence}

Aortic valve cryosections $(4.5 \mu \mathrm{m})$ were fixed with $4 \%$ paraformaldehyde for $15 \mathrm{~min}$ at room temperature. After blocking with $5 \%$ bovine serum albumin for $60 \mathrm{~min}$, the slides were immunostained with primary antibodies against CD11c (1:150; Abcam, Cat. No. EP1347Y), CD206 (1:150; Abcam, Cat. No. ab8918), IL-37 (1:150; Abcam, Cat. No. ab153889). The slides were then stained with a Cy3- or FITC-labeled secondary antibody (Beyotime Biotechnology), and mounted with Vectashield antifade mounting media (Beyotime Biotechnology). Negative controls were performed by incubation with secondary antibody alone (omitting primary antibody). Nuclei were stained with Hoechst 33258 (Beyotime Biotechnology), cell membranes were stained with 3,3'-dioctadecyloxacarbocyanine perchlorate (DiO) (Beyotime Biotechnology) according to manufacturer's instructions. Images were captured using fluorescence microscopy (Leica TCS-SP8 confocal microscope).

\section{Statistical Analysis}

All data are presented as mean \pm standard (SD). Statistical analysis was performed using SPSS 19.0 software. An unpaired, two-tailed Student's $t$-test was used for two-group comparisons. A one-way analysis of variance (ANOVA) or two-way ANOVA with the SNK/Dunnett test was used to analyze the statistical significance among multiple groups. A $P$ value $<0.05$ was considered statistically significant $\left({ }^{*} P<0.05\right.$, $\left.{ }^{* *} P<0.01,{ }^{* * *} P<0.001\right)$.

\section{RESULTS}

\section{More M1 Macrophages Infiltrated in Calcified Aortic Valves, Accompanied by a Reduction in IL-37 Levels}

Calcified aortic valves were collected from patients undergoing aortic valve replacement and normal aortic valves from patients undergoing heart transplantation surgery. Then the valves were stained with Hematoxylin and eosin (HE). The calcified valves showed marked fibrosis and neovascularization compared with the non-calcified valves (Figure 1A), which indicates a progression of tissue injury and fibrosis repair in CAVD. To detect M1 and M2 macrophage infiltration in the non-calcified and calcific valves, immunohistochemical staining was performed for CD11c (surface marker of M1) (Figure 1B) and CD206 (surface marker of M2) (Figure 1C). The infiltration of M1 macrophages (CD11c+) increased in calcified aortic valves $(P<0.01)$, while the accumulation of M2 macrophages $(\mathrm{CD} 206+)$ of that decreased $(P<0.05)$ (Figure 1E). Moreover, calcified aortic valves showed more CD11c+/iNOS cells (M1 macrophages) infiltration than CD206+ cells (M2 macrophages), while non-calcified aortic valves showed the opposite (Supplementary Figures S1A, S2A). And the ratio of CD11c+/CD206+ cells evidently increased in calcified aortic valves compared with non-calcified aortic values $(P<0.01)$ (Figure 1F). In non-calcified aortic valves, IL-37 expressed both intracellularly and extracellularly (Supplementary Figure S1B). Valve tissues were also stained with the anti-IL-37 antibody (Figure 1D), showing that IL-37 expression decreased in calcified aortic valves (Figure 1E). Immunoblots of noncalcified and calcified aortic valve tissues also showed the same trend (Supplementary Figure S2B). These findings suggested that IL-37 may inhibit M1 but augment M2 infiltration. These results demonstrated that M1 macrophage levels increased in 

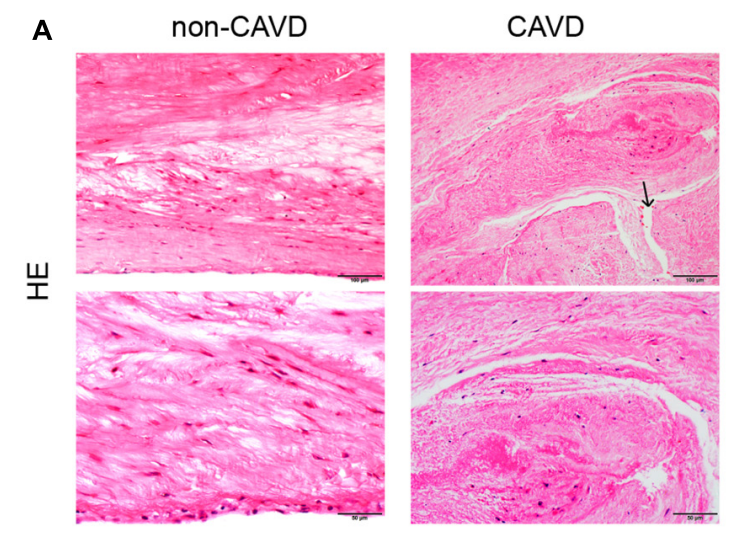

C
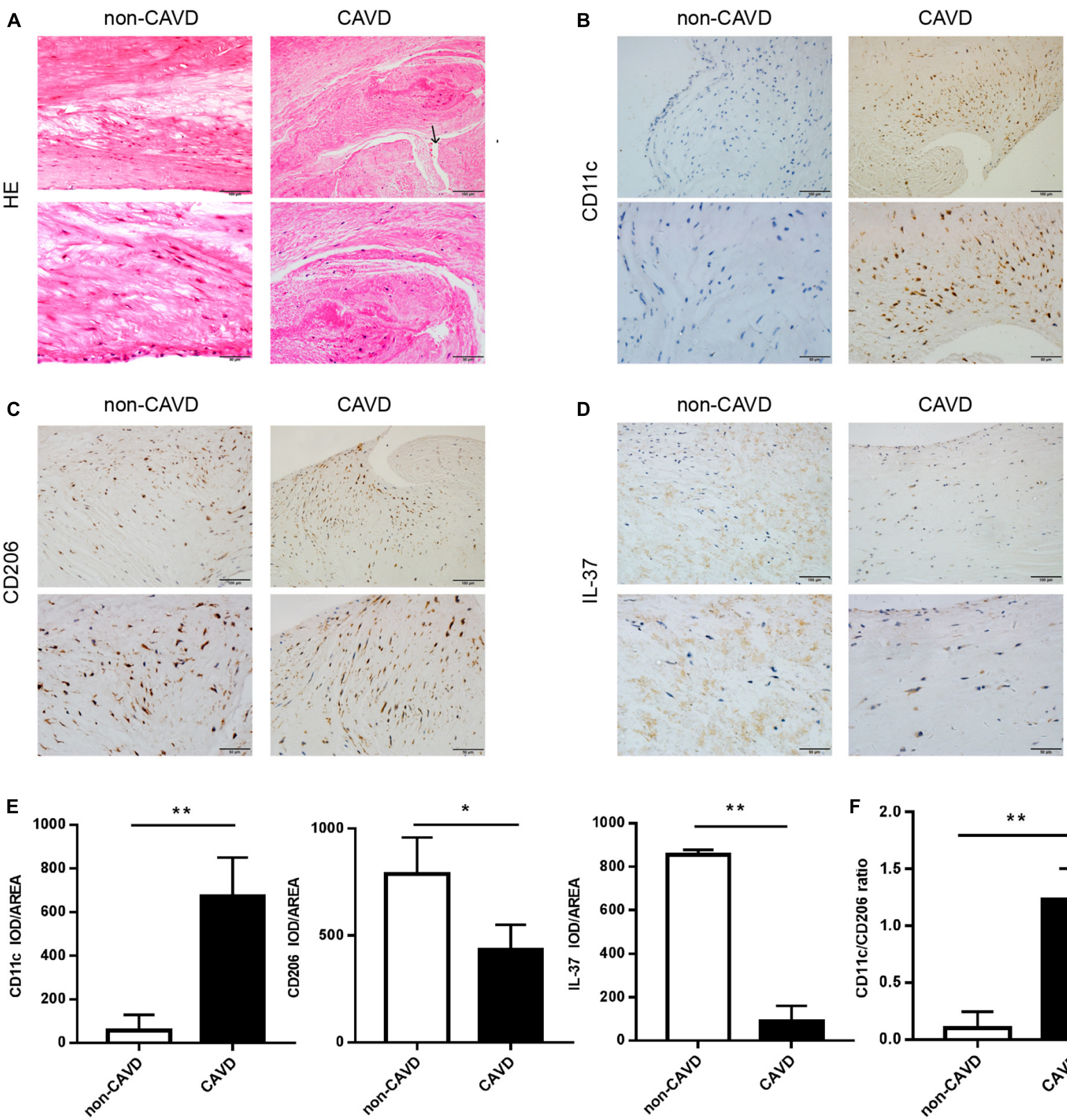

D
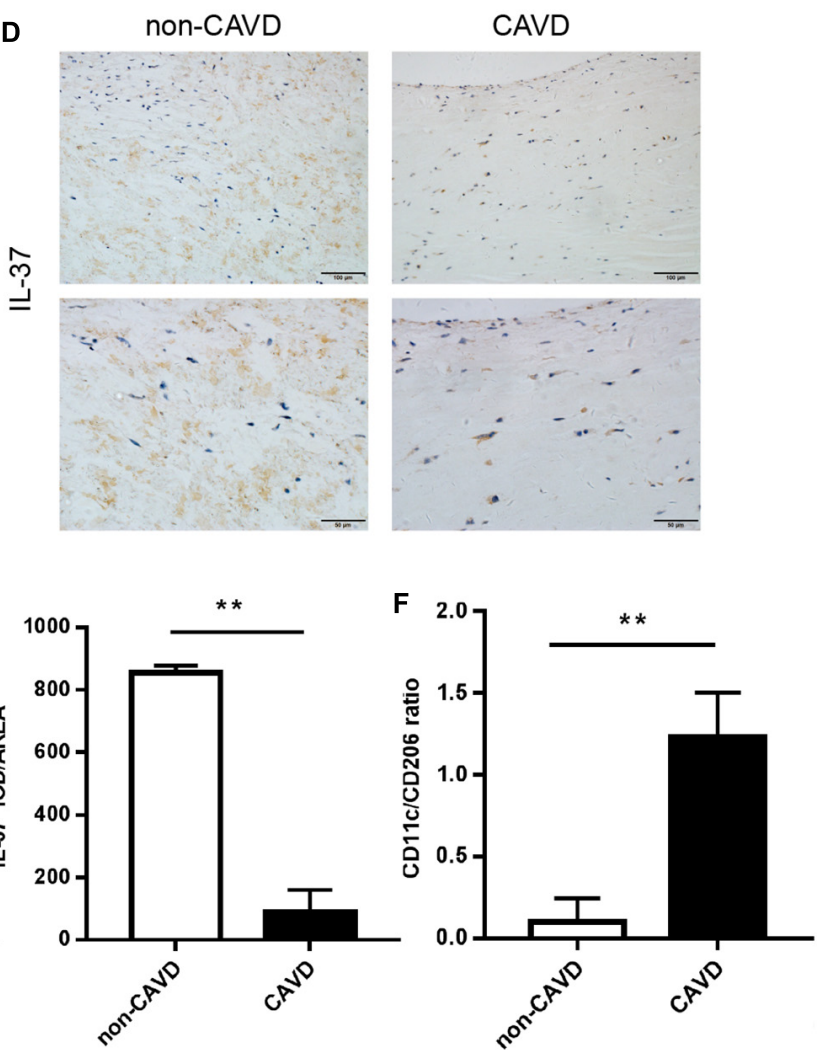

FIGURE 1 | More M1 macrophages infiltrate in calcified aortic valves, with a reduction of IL-37 expression. (A) Normal and calcified human aortic valves were stained with HE. The arrow above shows the newly blood vessels in calcified aortic valves. Magnification, $200 \times$ and $400 \times ; n=6$. (B-D) CD11C, CD206 and IL-37 expression are shown in calcified and non-calcified aortic valves. Magnification, 200× and 400×; $n=6$. (E) Integrated optical density (IOD)/area of CD11C, CD206 and IL-37. Magnification, 400x, scale bar $=50 \mu \mathrm{m}$. (F) CD11c + cell-to-CD206 + cell ratio in CAVD and non-CAVD. Data are presented as the mean \pm SD $(n=6)$; ${ }^{\star} P<0.05,{ }^{* *} P<0.01$. HE, hematoxylin-eosin; CAVD, calcific aortic valve disease; non-CAVD, non-calcific aortic valve disease.

calcified human aortic valves compared with non-calcified aortic valves, which may be associated with a reduction in IL-37 levels.

\section{IL-37 Down-Regulated M1 Phenotype Markers and Pro-inflammatory Cytokine Levels}

To determine whether IL-37 suppresses M1 macrophage differentiation, we treated Thp- 1 cells with PMA (100 ng/ml) to differentiate into resting (M0) macrophages, and then PMAtreated THP-1 cells were treated with LPS $(100 \mathrm{ng} / \mathrm{ml})$ and IFN- $\gamma(20 \mathrm{ng} / \mathrm{ml})$ in the presence or absence of recombinant IL-37. The levels of iNOS and CD11c increased significantly after treatment with LPS and IFN- $\gamma$. However, IL-37 markedly attenuated iNOS (Figure 2A) and CD11c (Figure 2B) expression in M1 macrophages. In addition, the levels of the pro-inflammatory cytokines MCP-1 and IL- 6 decreased in the cell lysate (Figures 2C,D) and the supernatant (Figures 2E,F) 

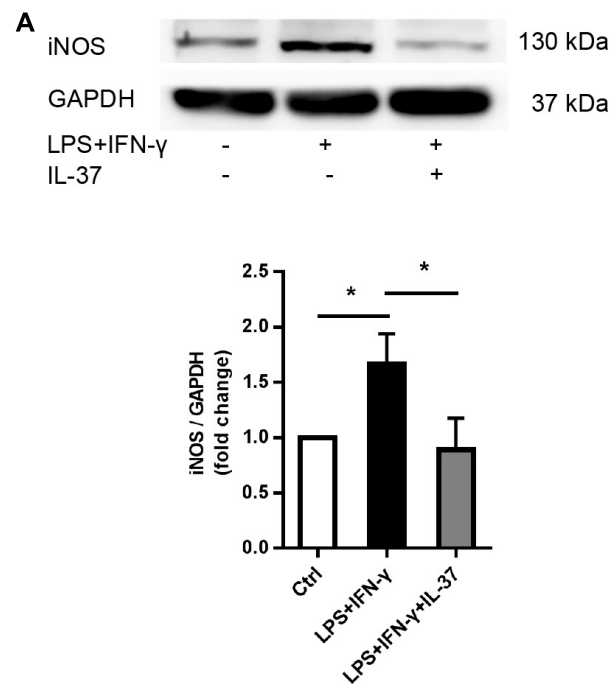

C

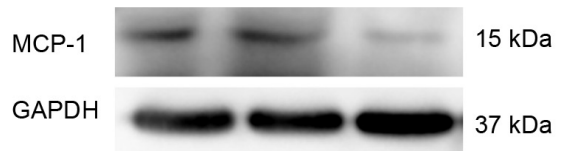

$\begin{array}{llll}\text { LPS+IFN-Y } & - & + & + \\ \mathrm{IL}-37 & - & - & +\end{array}$

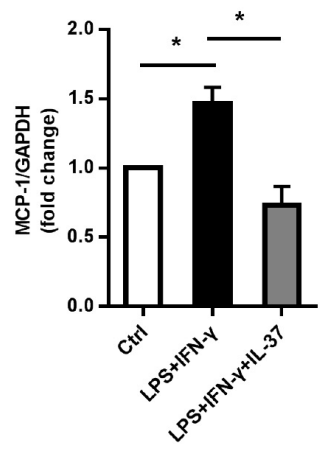

E

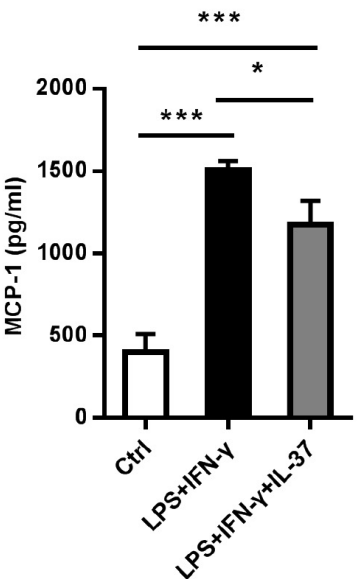

B
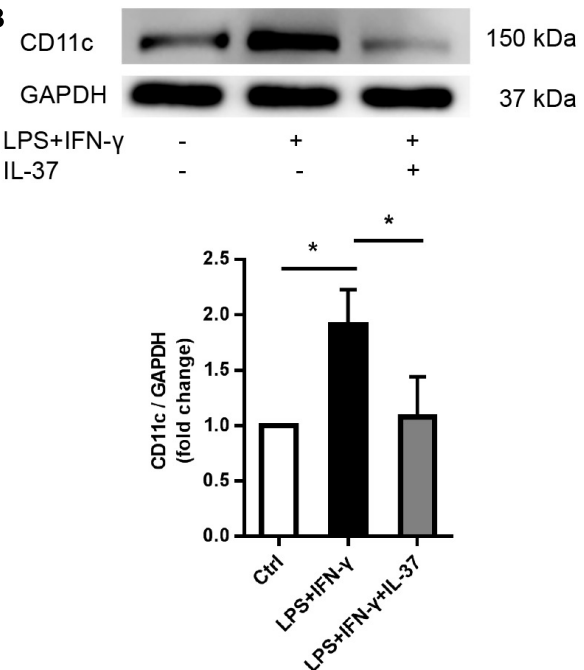

D
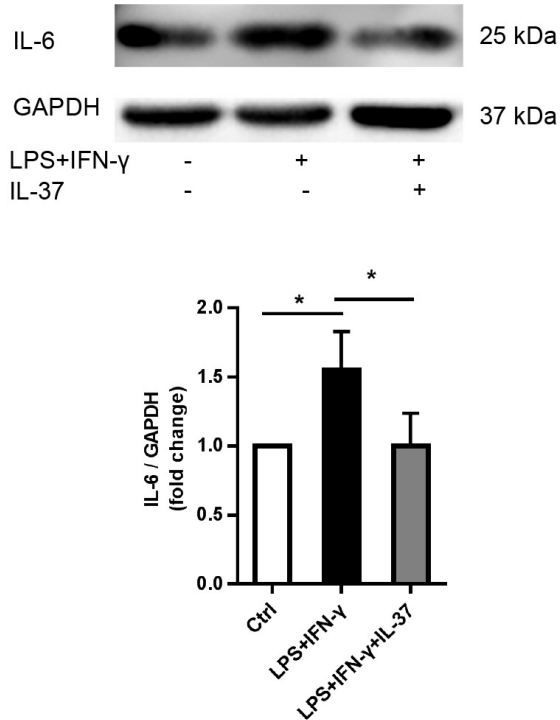

F

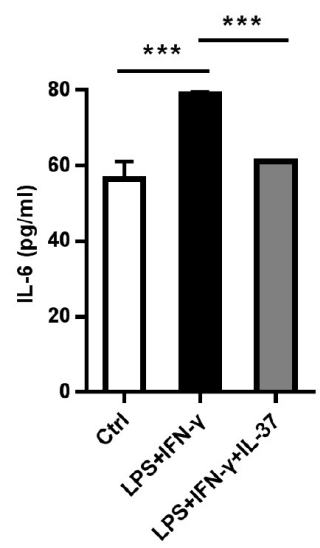

FIGURE 2 | IL-37 suppresses M1 macrophage polarization and pro-inflammatory cytokines secretion. PMA-treated THP-1 cells were treated with LPS (100 ng/ml) and IFN- $\gamma(20 \mathrm{ng} / \mathrm{ml})$ in the presence or absence of recombinant IL-37 $(0.1 \mathrm{ng} / \mathrm{ml})$. (A,B) Representative immunoblots and densitometric data show that IL-37 inhibits iNOS and CD11c expression; $n=3,{ }^{*} P<0.05$. (C,D) Representative immunoblots and densitometric data show that IL-37 down-regulates MCP-1 and IL-6 expression; $n=3,{ }^{\star} P<0.05$. (E,F) The ELISA data show that IL-37 down-regulates MCP-1 and IL-6 secretion; $n=3,{ }^{\star} P<0.05,{ }^{\star \star \star} P<0.001$. 
A
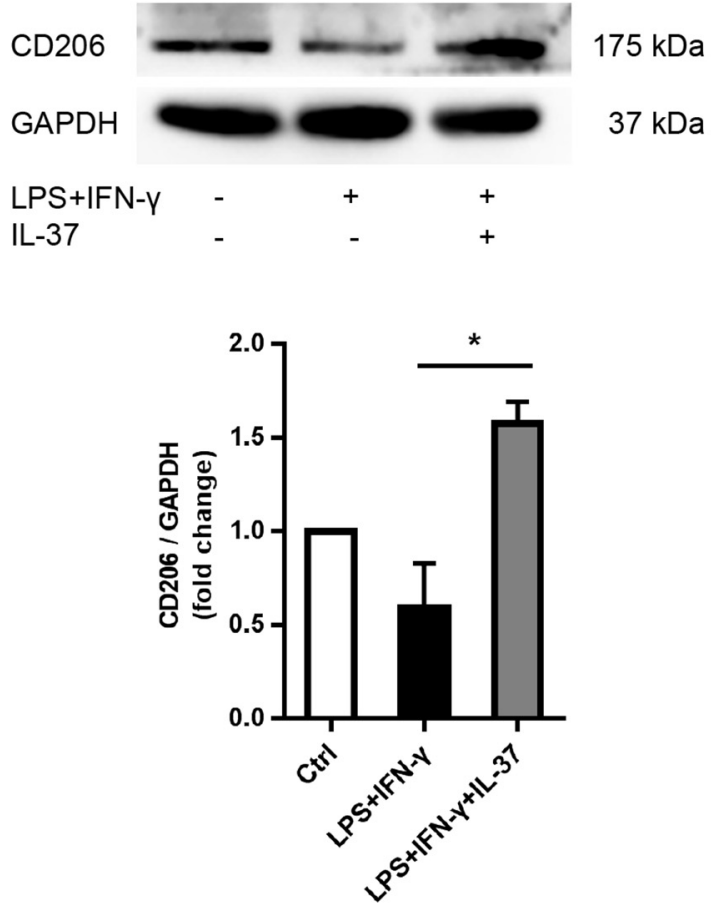

B

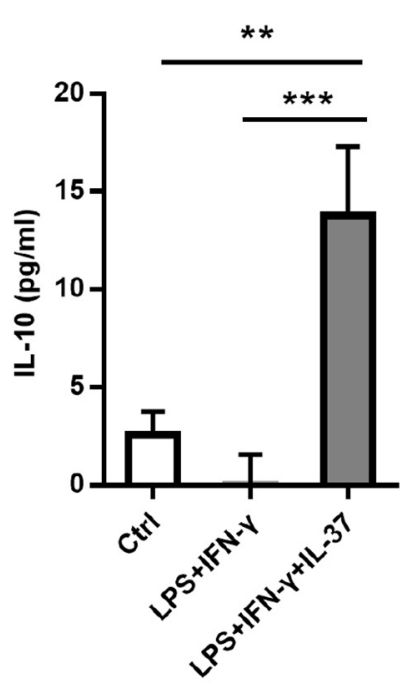

FIGURE 3 | IL-37 promotes the expression of M2 macrophage markers.(A) Representative immunoblots and densitometric data show that CD206 expression increases after pre-treating PMA-treated THP-1 cells with recombinant human IL-37 (0.1 ng/ml) prior to stimulating with LPS (100 ng/ml) and IFN- $\gamma(20 \mathrm{ng} / \mathrm{ml}) ; n=3$, ${ }^{\star} P<0.05$. (B) The ELISA data show that IL-37 up-regulates IL-10 secretion; $n=4,{ }^{\star \star} P<0.01,{ }^{\star \star \star} P<0.001$.

after treatment with IL-37 in M1 macrophages. Taken together, these results demonstrate that IL-37 inhibits the differentiation of macrophages into the M1 phenotype and attenuates inflammation.

\section{IL-37 Up-Regulated M2 Phenotype Marker Expression}

To determine the role of IL-37 in M2 polarization, we analyzed the cellular levels of the M2 macrophage markers CD206 and IL-10. PMA-treated THP-1 cells expressed less CD206 after exposed to LPS and IFN- $\gamma$. However, IL-37 increased CD206 expression (Figure 3A). ELISA showed that the levels of IL-10, an anti-inflammatory factor, were higher in the supernatant in the groups challenged by IL-37. However, the levels of IL-10 were very low in the group treated with LPS and IFN- $\gamma$ without IL37 (Figure 3B). These results indicated that IL-37 may somehow induce macrophages to differentiate into M2 macrophages.

\section{IL-37 Suppressed M1 Macrophage Polarization Through the NF- $\mathrm{B}$ Pathway}

To elucidate the mechanism by which IL-37 suppresses M1 macrophage polarization, we detected the activation of NF- $\mathrm{B}$, an important signaling molecule that regulates macrophage polarization. We stimulated PMA-treated THP-1 cells with LPS and IFN $-\gamma$ for $0-24 \mathrm{~h}$ and then examined NF- $\kappa$ B phosphorylation. As shown in Figure 4A, NF- $\kappa$ B p65 phosphorylation was markedly enhanced at $0.5 \mathrm{~h}$. Interestingly, evidently enhanced
NF- $\kappa \mathrm{B}$ p65 phosphorylation was also detected at 4,8 , and $24 \mathrm{~h}$ but not at $1 \mathrm{~h}$ or $2 \mathrm{~h}$. To further confirm the role of NF- $\kappa \mathrm{B}$ in the modulation of M1 macrophage polarization, we pre-treated PMA-treated THP-1 cells with the NF- $\kappa$ B inhibitor BAY11-7082 an hour prior to treatment with LPS and IFN- $\gamma$ for $24 \mathrm{~h}$. As shown in Figure 4B, iNOS expression decreased after treatment with BAY11-7082. In the following study, we stimulated PMAtreated THP-1 cells with LPS and IFN- $\gamma$ in the presence or absence of recombinant IL-37. We found that recombinant IL-37 reduced NF- $\kappa \mathrm{B}$ phosphorylation at different time point except at $0.5 \mathrm{~h}$ (Figure 4C and Supplementary Figure S3). These observations imply that IL-37 could suppress M1 polarization through inhibiting the NF- $\mathrm{B}$ pathway.

\section{IL-37 Modulated M1 Macrophage Polarization Through the Notch1 Signaling Pathway and Thus Modulated NF-кB Pathway Activation}

As shown in Figure 5A, LPS and IFN- $\gamma$ co-treatment obviously induced the upregulation of Notch1 intracellular domain (NICD1) after $4 \mathrm{~h}$. To determine whether the Notch1 signaling pathway modulates M1 macrophage polarization, we used DAPT, a $\gamma$-secretase inhibitor, to inhibit Notch1 intracellular domain (NICD1) generation (Figure 5B). Interestingly, treatment with DAPT evidently reduced iNOS levels in M1 macrophages (Figure 5C), indicating that the Notch1 pathway plays an important role in mediating M1 macrophage polarization. 
A
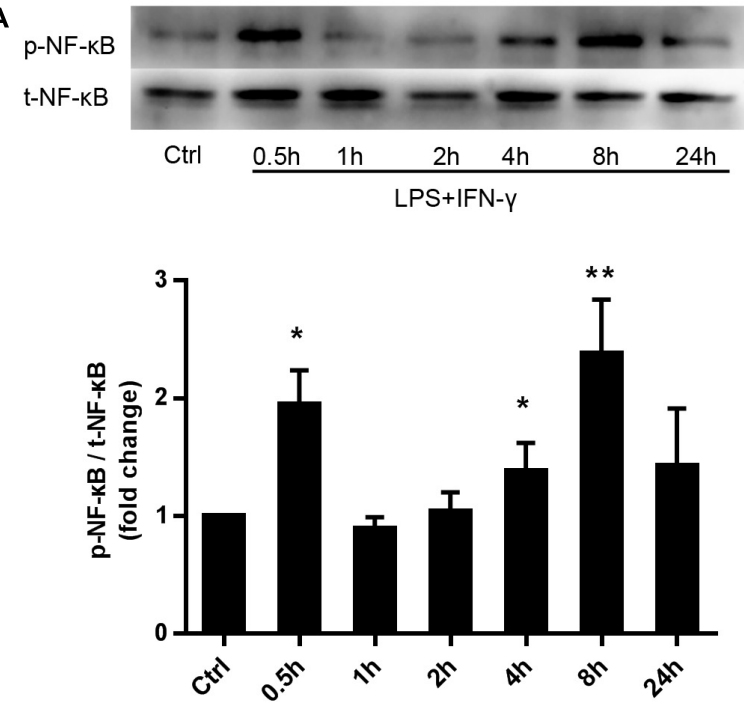

C $\mathrm{p}-\mathrm{NF}-\mathrm{kB}$
$\mathrm{t}-\mathrm{NF}-\mathrm{kB}$

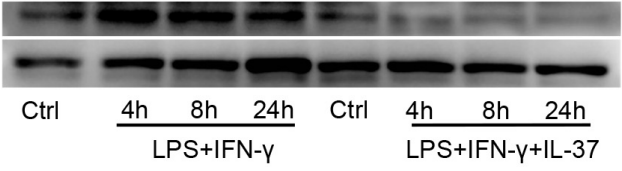

$65 \mathrm{kDa}$ $65 \mathrm{kDa}$

B

$65 \mathrm{kDa}$

$65 \mathrm{kDa}$

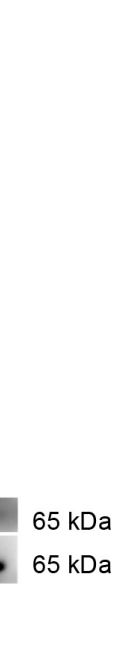
iNOS GAPDH

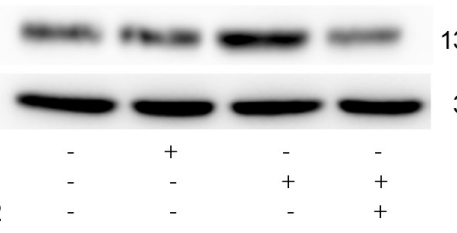

$130 \mathrm{kDa}$

DMSO

LPS+IFN-Y BAY11-7082

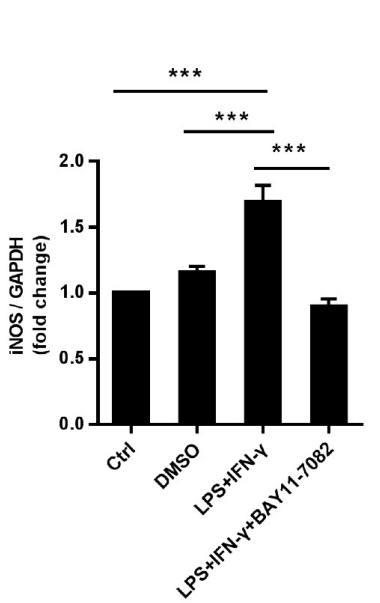

$37 \mathrm{kDa}$

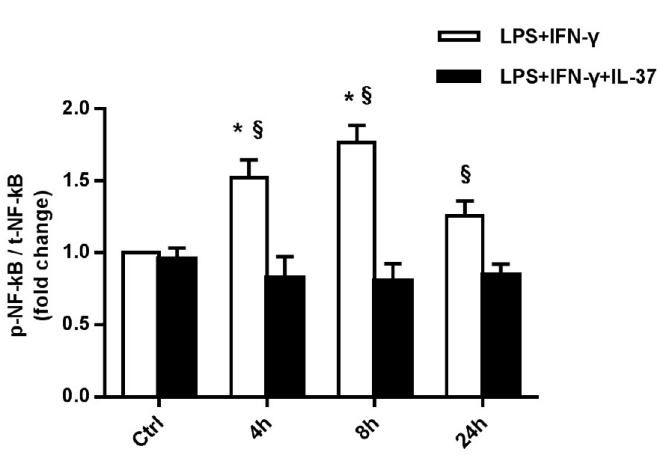

FIGURE 4 | IL-37 suppresses M1 polarization through the NF-kB signaling pathway. (A) Representative immunoblots and densitometric data show that PMA-treated THP-1 cells exhibit enhanced NF-kB phosphorylation after treatment with LPS (100 ng/ml) and IFN- $\gamma(20 \mathrm{ng} / \mathrm{ml})$ for 0.5 to $24 \mathrm{~h} ; n=3,{ }^{*} P<0.05$, ${ }^{\star \star} P<0.01 \mathrm{vs}$. corresponding control. (B) PMA-treated THP-1 cells were treated with BAY11-7082 $(5 \mu \mathrm{M}) 1 \mathrm{~h}$ prior to being treated with LPS $(100 \mathrm{ng} / \mathrm{ml})$ and IFN- $\gamma(20 \mathrm{ng} / \mathrm{ml})$. Representative immunoblots and densitometric data show that NF-kB pathway inhibition decreases iNOS expression in M1 macrophages; $n=3,{ }^{\star \star \star} P<0.001$. (C) PMA-treated THP-1 cells were treated with LPS (100 ng/ml) and IFN- $\gamma(20 \mathrm{ng} / \mathrm{ml})$ for $4-24 \mathrm{~h}$ in the presence or absence of recombinant IL-37 (0.1 ng/ml). Representative immunoblots and densitometric data show that treatment with recombinant IL-37 results in a reduction in NF-kB phosphorylation at different time point; $n=3,{ }^{*} P<0.05$ vs. corresponding control, $\S P<0.05$ vs. M1 macrophages treated with IL-37.

To further explore whether IL-37 suppresses M1 macrophage polarization through the Notch1 pathway, we treated PMAtreated THP-1 cells with recombinant IL-37 $(0.1 \mathrm{ng} / \mathrm{ml}) 1 \mathrm{~h}$ prior co-treatment with LPS and IFN- $\gamma$ for $4-24 \mathrm{~h}$. The immunoblot showed that IL-37 decreased Notch1 pathway activation at different time points and markedly reduced NICD1 production (Figure 5D). These findings indicate that IL-37 could modulate M1 macrophage polarization through Notch1 pathway.

Our previous study have shown that Notch1 modulates the inflammatory response in the interstitial cells of human aortic valves through LPS-induced NF- $\kappa$ B pathway (Zeng et al., 2012).
To determine the role of Notch 1 in NF- $\kappa \mathrm{B}$ activation, we treated PMA-treated THP-1 cells with DAPT before co-treatment with LPS and IFN- $\gamma$. NF- $\kappa$ B phosphorylation was significantly reduced by Notch1 pathway inhibition (Figure 5E). In addition, as shown in Figures $\mathbf{4 A}, \mathbf{5 A}$, after treatment with LPS and IFN $-\gamma$, NF- $\kappa$ B activation was consistent with Notch1 activation from 4-24 h. These evidences indicated that the Notch1 signaling pathway modulates NF- $\kappa \mathrm{B}$ pathway activation to regulate M1 macrophage polarization. Therefore, IL-37 could decrease NF- $\mathrm{B}$ activation through suppressing the Notch1 pathway. 
A
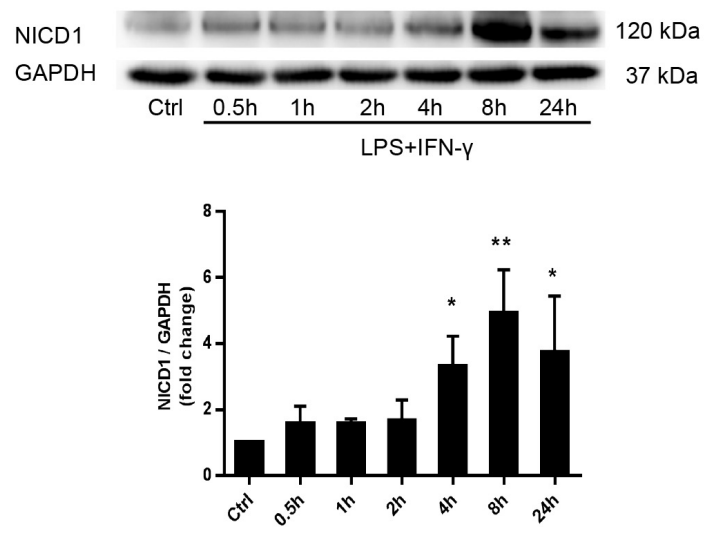

B

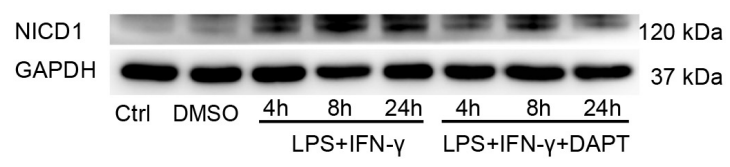

D
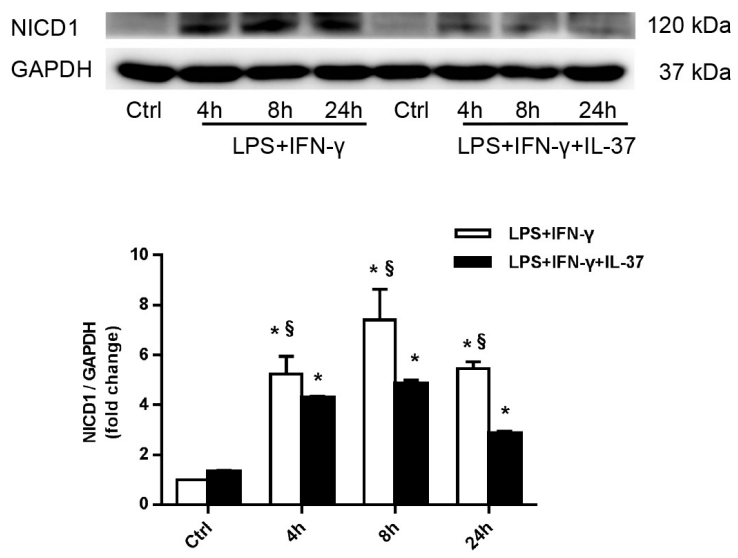

C

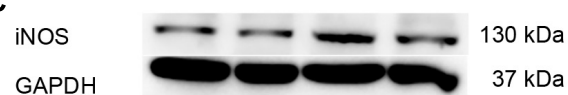

GAPDH

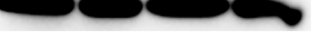

$37 \mathrm{kDa}$

DMSO LPS+IFN-Y $\quad-\quad+\quad-\quad+$ DAPT

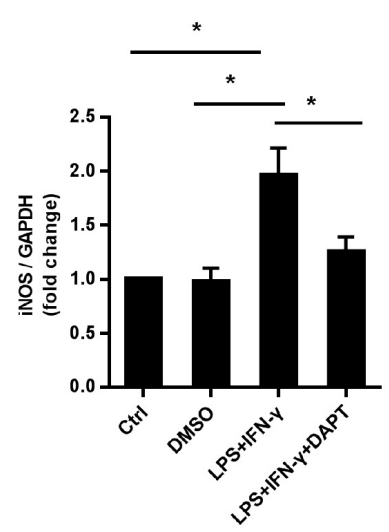

E

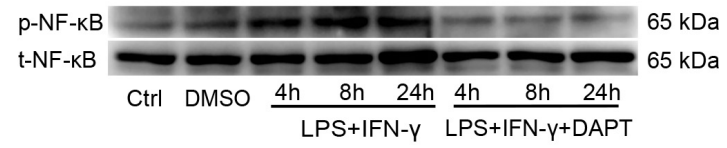

FIGURE 5 | IL-37 modulates macrophage polarization through suppressing activation of the Notch1- NF- $\mathrm{B}$ pathway axis. (A) Representative immunoblots and densitometric data show that stimulation with LPS and IFN- $\gamma$ enhances Notch1 pathway activation in PMA-treated THP-1 cells; $n=3$, ${ }^{\star} P<0.05$, ${ }^{\star \star} P<0.01$. (B,C) PMA-treated THP-1 cells were treated with DAPT (50 $\mu \mathrm{M}) 1 \mathrm{~h}$ prior to LPS (100 ng/ml) and IFN- $\gamma(20 \mathrm{ng} / \mathrm{ml})$ for 4-24 h. (B) Representative immunoblots show that DAPT inhibits Notch1 activation; $n=3$. (C) Representative immunoblots and densitometric data show that Notch1 pathway inhibition reduces iNOS expression in M1 macrophages; $n=3$, * $P<0.05$. (D) PMA-treated THP-1 cells were treated with recombinant human IL-37 (0.1 ng/ml) $1 \mathrm{~h}$ prior to being treated with LPS $(100 \mathrm{ng} / \mathrm{ml})$ and IFN- $\gamma(20 \mathrm{ng} / \mathrm{ml})$ at different times. Representative immunoblots and densitometric data show that IL-37 decreases NICD1 expression at different time point in M1 macrophages; $n=3$. ${ }^{\star} P<0.05$ vs. corresponding control, $\S P<0.05$ vs. M1 macrophages treated with IL-37. (E) Representative immunoblots show that Notch1 pathway inhibition decreases NF-kB phosphorylation; $n=3$.

\section{DISCUSSION}

Chronic inflammation and osteogenic activity in the aortic valve leaflet tissue contribute to CAVD (Rajamannan et al., 2011; Natorska and Undas, 2015). Infiltration of macrophages, particularly M1 macrophages, promotes CAVD mainly through the cross-talk with aortic valve interstitial cells (AVICs) (Lee and Choi, 2016). The present study uncovered an antiinflammation function of IL-37 through suppression of M1 macrophages. IL-37, expressed in human but not in murine, has been proved possessing an anti-inflammatory potential in innate and adaptive immunity both in vitro and in vivo (Nold et al., 2010).

In the present study, we demonstrated that calcific aortic valves showed more M1 but less M2 macrophage infiltration with deficiency in IL-37, compared with normal aortic valves. Notably, recombinant human IL-37 suppressed M1 polarization and expression of pro-inflammatory cytokines. Further, differentiating M1 macrophages into M2 macrophages may be induced by the micro environment created by IL-37. Mechanistic evidences showed that IL-37 attenuated M1 polarization through the inhibition of Notch1 and NF-кB activation, the pathways 
that regulate the polarization of macrophages (Wang Y. C. et al., 2010; Adamson et al., 2016). It should be noted that the Notch1$\mathrm{NF}-\kappa \mathrm{B}$ axis activation was involved in M1 polarization, which could be suppressed by IL-37.

Here, we showed that calcified human aortic valves expressed lower levels of IL-37 protein and that this alteration in cellular IL-37 levels might be associated with the more infiltration of M1 macrophages but less of M2 in diseased aortic valves. Staining of Hematoxylin and eosin showed that the formation of new blood vessels promoted and the elastic fibers were in degeneration and disorder in diseased valves. Further, the ratio of M1 to M2 macrophages markedly increased in calcified aortic valves, with a lower expression of IL-37, indicating that levels of IL-37 in aortic valves may play a role in macrophage polarization. In vitro experiments, treatment with IL-37 effectively suppressed M1 macrophage polarization and thus reduced the expression of pro-inflammatory cytokines, such as IL-6 and MCP-1. However, M2 macrophages and its secreting anti-inflammatory cytokine IL-10 increased when exposed to recombinant IL-37. These lines of evidences suggest that IL-37 plays an important role in suppression of M1 macrophage polarization and may promote the differentiation of M2 macrophages.

IL-37 suppresses M1 macrophage polarization and thus exerts anti-inflammatory effect, which may attenuate the calcified process of CAVD. CAVD is a slowly progressing disorder characterized by lipoprotein deposition, chronic inflammation, and active leaflet calcification (Freeman and Otto, 2005). In normal valves, aortic valvular endothelial cells (AVECs) and aortic valvular interstitial cells (AVICS) are important to maintain the homeostasis in the aortic valves (Combs and Yutzey, 2009). Impairment of valvular endothelium due to damage to AVECs can result in accumulation of lipoproteins (LDL) in the subendothelial space. Oxidized LDL (oxLDL), derived from LDL molecules, can trigger macrophage infiltration into aortic valves by increasing cell adhesion molecules, such as ICAM-1 and VCAM-1 (Mohty et al., 2008). These infiltrating macrophages release pro-inflammatory cytokines, including IL6 and TNF- $\alpha$, which differentiate AVECs into AVICs via endothelial-mesenchymal transition (Mahler et al., 2013; Lee and Choi, 2016). AVICs, the most abundant cell type in aortic valves, are capable of differentiating into myofibroblasts and osteoblastlike cells in specific conditions, which promote fibrosis and valve calcification, respectively (Rajamannan et al., 2011; Lee and Choi, 2016). Osteoblast-like AVICs promote aortic calcification through a mechanism similar to osteogenesis (Rajamannan et al., 2003). In calcified aortic valves, more monocytes infiltrate into the tissue to differentiate into pro-inflammatory macrophages, which secret inflammatory cytokines such as IL-6 and TNF- $\alpha$, promoting AVICs activation and inducing alkaline phosphatase expression, eventually leading to aortic valvular calcification (Kaden et al., 2005; Lee and Choi, 2016). Thus, through reducing the inflammatory cytokines produced by M1 macrophages, IL-37 may inhibit the osteogenic activity of AVICs and therefore alleviate the calcification.

Here, we demonstrated that IL-37 suppresses M1 polarization and may convert M1 into M2 macrophages. Recombinant human IL-37 suppressed the switch response to the TLR4 agonist (LPS) and IFN- $\gamma$, which inhibits PMA-treated THP-1 cells to polarize into M1 macrophages. In CAVD, M1 macrophages play a proinflammatory role, with the expression of inflammatory cytokines including MCP-1, IL-6 and TNF- $\alpha$ whereas M2 macrophages play an anti-inflammatory role, secreting anti-inflammatory cytokine IL-10 (Lawrence and Natoli, 2011; Mantovani et al., 2013; Liu et al., 2014). It has been demonstrated that macrophages exhibit functional plasticity and M1 and M2 macrophages transform into each other as a result of changes in the microenvironment in infectious diseases, atherosclerosis and calcific aortic diseases (Gordon and Martinez, 2010; Liu et al., 2014). Notably, a recent research reports that IL-37 directly suppresses M1 polarization in Concanavalin A induced liver injury C57BL/6 mice while promotes M2 polarization indirectly by increasing the expression of IL-4 and IL-13 (Feng et al., 2019). In this study, we showed that IL-37 attenuated the inflammation induced by M1 macrophages and may convert M1 into M2 macrophages, and thus had a potential to dampen the calcification of CAVD.

Several studies have demonstrated that the Notch1 and NF- $\kappa \mathrm{B}$ pathways induce macrophages to differentiate into M1 (Palaga et al., 2008; Wang Y. et al., 2010; Zhang et al., 2015; An et al., 2017). To investigate the effects of TLR on Notch receptor expression, the researchers treats the macrophage-like cell line RAW264.7 with LPS, IFN- $\gamma$ or LPS plus IFN- $\gamma$. These treatments result in the significant upregulation of Notch1 transcription. Treatment by LPS plus IFN- $\gamma$ is the strongest inducer of the up-regulation of Notch1 transcription (Palaga et al., 2008). The forced activation of Notch signaling augments M1 polarization regardless of whether

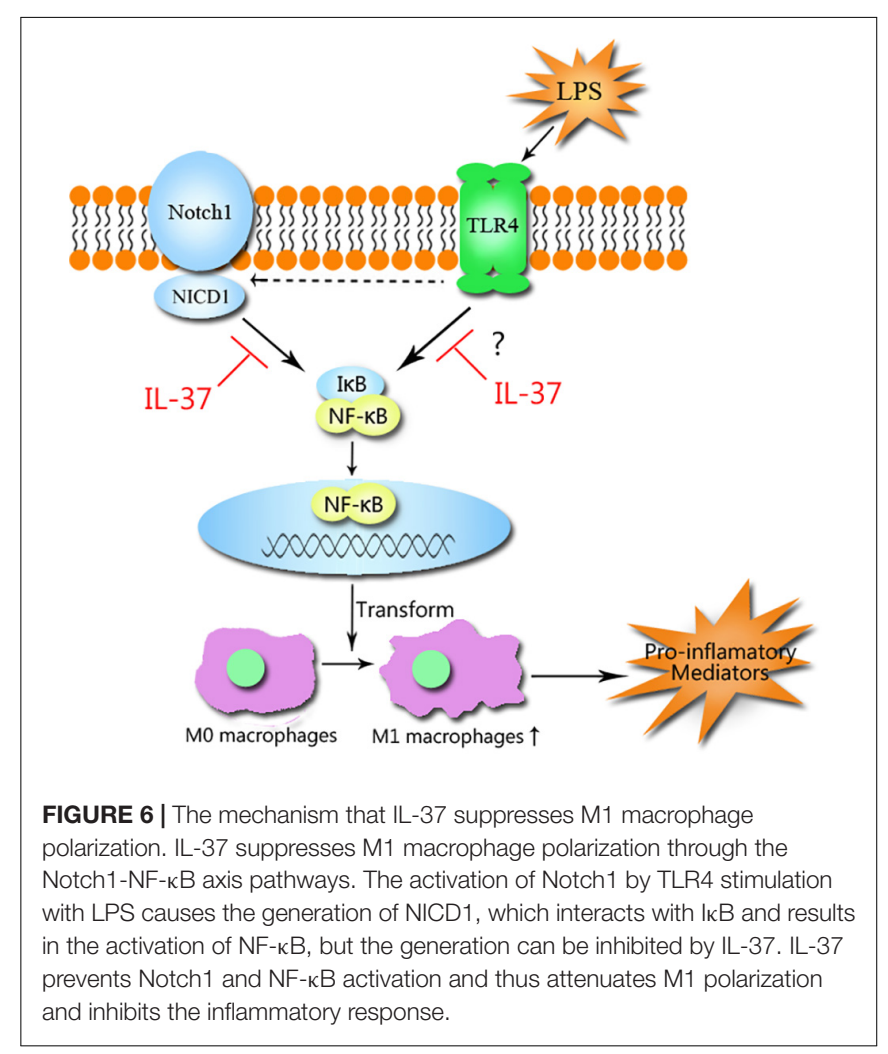


M1 or M2 inducers are applied. Inhibition of Notch signaling causes M2 polarization even in the presence of M1 inducers (Wang Y. et al., 2010). It seems that the Notch signaling pathway is essential for M1 polarization.

In this study, we noted that recombinant human IL-37 suppressed M1 polarization by inhibiting NF- $\mathrm{B}$ phosphorylation and Notch1 activation. Interestingly, suppression of Notch1 activation evidently decreased NF- $\kappa$ B pathway phosphorylation, indicating that the Notch1 pathway modulated NF- $\kappa \mathrm{B}$ activation. Our previous study showed that recombinant human IL-37 suppresses NF- $\kappa$ B phosphorylation and inhibits ERK1/2 phosphorylation in AVICs exposed to LPS, Pam3CSK4, or oxLDL (Zeng et al., 2017). Notch1 modulates NF$\kappa \mathrm{B}$ activation to mediate complex responses in both macrophages and AVICs (Palaga et al., 2008; Zeng et al., 2013). Thus, inhibiting Notch1 with IL-37 alleviates Notch1 and NF- $\kappa$ B activation to efficiently suppress M1 polarization and attenuate inflammation.

Our previous study showed that stimulating TLR4 with LPS induces the release of Jagged1, a Notch1 ligand. Activation of Notch1 by Jagged1 causes the generation of NICD1, which enhances NF- $\mathrm{KB}$ activation by interacting with IKK, resulting in the augmentation of the inflammatory response (Zeng et al., 2012). Activation of Notch signaling augments M1 polarization, while inhibition of the signaling way increases M2 polarization (Wang Y. et al., 2010). Based on these findings and the evidence collected by this study, we demonstrate that IL-37 suppresses M1 polarization mainly by inhibiting the Notch1- NF- $\kappa$ B axis activation (Figure 6). However, whether IL-37 attenuates M1 polarization directly by inhibiting NF- $\kappa \mathrm{B}$ activation deserves further investigation.

Notably, IL-37 exerts anti-inflammatory function through both extracellular and intracellular pathways. To exert antiinflammatory effects, extracellular IL-37 binds to IL-18R $\alpha$ and then the co-receptor IL-1R8 (formerly SIGIRR) is recruited to form the tripartite ligand-receptor complex IL-37-IL-1R8-IL$18 \mathrm{R} \alpha$ to suppress NF- $\kappa \mathrm{B}$ function (Lunding et al., 2015). Also, the complex augments the inhibition of the production of IFN- $\gamma$ and thus suppresses M1 polarization (Nold et al., 2010; NoldPetry et al., 2015). IL-37 triggers inhibition of several signaling pathways, such as the Notch1, NF- $\mathrm{B}, \mathrm{mTOR}$, and MAPK pathways, thereby alleviating M1 polarization. However, the protein induces an increase in the activity of anti-inflammatory pathways, such as the STAT6, Mer and AMPK pathways, resulting in M2 polarization (O'Neill and Hardie, 2013; NoldPetry et al., 2015). Meanwhile, IL-37 translocates to the nucleus after caspase- 1 processing and interacts with the transcription factor Smad3 in LPS-stimulated macrophages to exert its antiinflammatory effects (Nold et al., 2010; Garlanda et al., 2013). Thus, we assume that IL-37 exerts an influence on these signaling pathways in macrophages to modulate polarization and attenuate inflammation.

The present study has limitations. In this study, we focused on the regulation of IL-37 on M1 polarization of macrophages. However, whether IL-37 could directly induce M2 macrophage polarization or switch M1 phenotype into M2 and its mechanism is still unclear. For further investigation, we will make a deeper research on the regulation of IL-37 in M2 polarization.

\section{CONCLUSION}

In summary, our study showed that calcific aortic valve leaflets exhibit a reduction in IL-37 and more M1 infiltration than normal aortic valves and thus exist in a pro-inflammatory state. The study also showed that recombinant IL-37 suppresses M1 macrophage polarization through Notch1-NF- $\kappa$ B axis pathway, thereby exerting a marked anti-inflammatory effect. Totally, these findings demonstrate that IL-37 has the potential to attenuate CAVD progression by suppressing M1 macrophage polarization.

\section{DATA AVAILABILITY STATEMENT}

All datasets generated for this study are included in the article/Supplementary Material.

\section{ETHICS STATEMENT}

The studies involving human participants were reviewed and approved by the Ethical Committee of Nanfang Hospital, China. The patients/participants provided their written informed consent to participate in this study.

\section{AUTHOR CONTRIBUTIONS}

PZ, SS, XY, SZ, XM, DZ, DX, and QZ conceived and designed the experiments. $\mathrm{PZ}, \mathrm{QL}, \mathrm{WD}, \mathrm{SZ}$, and $\mathrm{QM}$ performed the experiments. PZ, QL, and SZ analyzed the data. PZ, WD, SZ, DX, and QZ wrote the manuscript.

\section{FUNDING}

This project was partly supported by the National Natural Science Foundation of China (81570352 and 81770386), the Science and Technology Program of Guangzhou (201804010086), the Science and Technology Program of Guangdong Province (2017A050501019), and the Frontier Research Program of Guangzhou Regenerative Medicine and Health Guangdong Laboratory (2018GZR110105001).

\section{ACKNOWLEDGMENTS}

The authors thank Jiaying Li, Dingji Zhu, and Wenjun Xiong for their technical assistance.

\section{SUPPLEMENTARY MATERIAL}

The Supplementary Material for this article can be found online at: https://www.frontiersin.org/articles/10.3389/fcell.2020.00056/ full\#supplementary-material 


\section{REFERENCES}

Adamson, S. E., Griffiths, R., Moravec, R., Senthivinayagam, S., Montgomery, G., Chen, W., et al. (2016). Disabled homolog 2 controls macrophage phenotypic polarization and adipose tissue inflammation. J. Clin. Invest. 126, 1311-1322. doi: 10.1172/JCI79590

An, T., He, Q., Xia, Y., Chen, S., Baral, S., Mao, L., et al. (2017). MiR$181 \mathrm{~b}$ antagonizes atherosclerotic plaque vulnerability through modulating macrophage polarization by directly targeting notch1. Mol. Neurobiol. 54, 6329-6341. doi: 10.1007/s12035-016-0163-1

Boraschi, D., Lucchesi, D., Hainzl, S., Leitner, M., Maier, E., Mangelberger, D., et al. (2011). IL-37: a new anti-inflammatory cytokine of the IL-1 family. Eur. Cytokine Netw. 22, 127-147. doi: 10.1684/ecn.2011.0288

Combs, M. D., and Yutzey, K. E. (2009). Heart valve development. Circ. Res. 105, 408-421. doi: 10.1161/CIRCRESAHA.109.201566

Dinarello, C. A., and Bufler, P. (2013). Interleukin-37. Semin. Immunol. 25, 466468. doi: 10.1016/j.smim.2013.10.004

Dinarello, C. A., Nold-Petry, C., Nold, M., Fujita, M., Li, S., Kim, S., et al. (2016). Suppression of innate inflammation and immunity by interleukin-37. Eur. J. Immunol. 46, 1067-1081. doi: 10.1002/eji.201545828

Feng, X. X., Chi, G., Wang, H., Gao, Y., Chen, Q., Ru, Y. X., et al. (2019). IL37 suppresses the sustained hepatic IFN-gamma/TNF-alpha production and T cell-dependent liver injury. Int. Immunopharmacol. 69, 184-193. doi: 10.1016/ j.intimp.2019.01.037

Foldi, J., Shang, Y., Zhao, B., Ivashkiv, L. B., and Hu, X. (2016). RBP-J is required for M2 macrophage polarization in response to chitin and mediates expression of a subset of M2 genes. Protein Cell 7, 201-209. doi: 10.1007/s13238-0160248-7

Freeman, R. V., and Otto, C. M. (2005). Spectrum of calcific aortic valve disease: pathogenesis, disease progression, and treatment strategies. Circulation 111, 3316-3326. doi: 10.1161/circulationaha.104.486738

Garlanda, C., Dinarello, C. A., and Mantovani, A. (2013). The interleukin-1 family: back to the future. Immunity 39, 1003-1018. doi: 10.1016/j.immuni.2013. 11.010

Genin, M., Clement, F., Fattaccioli, A., Raes, M., and Michiels, C. (2015). M1 and M2 macrophages derived from THP-1 cells differentially modulate the response of cancer cells to etoposide. BMC Cancer 15:577. doi: 10.1186/s12885-0151546-9

Gordon, S., and Martinez, F. O. (2010). Alternative activation of macrophages: mechanism and functions. Immunity 32, 593-604. doi: 10.1016/j.immuni.2010. 05.007

Hjortnaes, J., Butcher, J., Figueiredo, J. L., Riccio, M., Kohler, R. H., Kozloff, K. M., et al. (2010). Arterial and aortic valve calcification inversely correlates with osteoporotic bone remodelling: a role for inflammation. Eur. Heart J. 31, 1975-1984. doi: 10.1093/eurheartj/ehq237

Kaden, J. J., Kilic, R., Sarikoc, A., Hagl, S., Lang, S., Hoffmann, U., et al. (2005). Tumor necrosis factor alpha promotes an osteoblast-like phenotype in human aortic valve myofibroblasts: a potential regulatory mechanism of valvular calcification. Int. J. Mol. Med. 16, 869-872.

Lawrence, T., and Natoli, G. (2011). Transcriptional regulation of macrophage polarization: enabling diversity with identity. Nat. Rev. Immunol. 11, 750-761. doi: $10.1038 /$ nri3088

Lee, S. H., and Choi, J. (2016). Involvement of immune cell network in aortic valve stenosis: communication between valvular interstitial cells and immune cells. Immune Netw. 16:26. doi: 10.4110/in.2016.16.1.26

Li, X. F., Wang, Y., Zheng, D. D., Xu, H. X., Wang, T., Pan, M., et al. (2016). M1 macrophages promote aortic valve calcification mediated by microRNA214/TWIST1 pathway in valvular interstitial cells. Am. J. Transl. Res. 8, 57735783.

Liu, L., Liang, L., Liang, H., Wang, M., Lu, B., Xue, M., et al. (2019). Fusobacterium nucleatum aggravates the progression of colitis by regulating M1 macrophage polarization via AKT2 pathway. Front. Immunol. 10:1324. doi: 10.3389/fimmu. 2019.01324

Liu, Y., Zou, X., Chai, Y., and Yao, Y. (2014). Macrophage polarization in inflammatory diseases. Int. J. Biol. Sci. 10, 520-529. doi: 10.7150/ijbs.8879

Lunding, L., Webering, S., Vock, C., Schroder, A., Raedler, D., Schaub, B., et al. (2015). IL-37 requires IL-18Ralpha and SIGIRR/IL-1R8 to diminish allergic airway inflammation in mice. Allergy 70, 366-373. doi: 10.1111/all.12566
Mahler, G. J., Farrar, E. J., and Butcher, J. T. (2013). Inflammatory cytokines promote mesenchymal transformation in embryonic and adult valve endothelial cells. Arterioscler. Thromb. Vasc. Biol. 33, 121-130. doi: 10.1161/ATVBAHA.112.300504

Mantovani, A., Biswas, S. K., Galdiero, M. R., Sica, A., and Locati, M. (2013). Macrophage plasticity and polarization in tissue repair and remodelling. J. Pathol. 229, 176-185. doi: 10.1002/path.4133

Mohler, E. R., Gannon, F., Reynolds, C., Zimmerman, R., Keane, M. G., and Kaplan, F. S. (2001). Bone formation and inflammation in cardiac valves. Circulation 103, 1522-1528. doi: 10.1161/01.cir.103.11.1522

Mohty, D., Pibarot, P., Després, J., Cote, C., Arsenault, B., Cartier, A., et al. (2008). Association between plasma LDL particle size, valvular accumulation of oxidized LDL, and inflammation in patients with aortic stenosis. Arterioscler. Thromb. Vasc. Biol. 28, 187-193. doi: 10.1161/atvbaha.107.154989

Natorska, J., and Undas, A. (2015). Blood coagulation and fibrinolysis in aortic valve stenosis: links with inflammation and calcification. Thromb. Haemostasis. 114:217. doi: 10.1160/TH14-10-0861

Nold, M. F., Nold-Petry, C. A., Zepp, J. A., Palmer, B. E., Bufler, P., and Dinarello, C. A. (2010). IL-37 is a fundamental inhibitor of innate immunity. Nat. Immunol. 11, 1014-1022. doi: 10.1038/ni.1944

Nold-Petry, C. A., Lo, C. Y., Rudloff, I., Elgass, K. D., Li, S., Gantier, M. P., et al. (2015). IL-37 requires the receptors IL-18R $\alpha$ and IL-1R8 (SIGIRR) to carry out its multifaceted anti-inflammatory program upon innate signal transduction. Nat. Immunol. 16, 354-365. doi: 10.1038/ni.3103

Odegaard, J. I., Ricardo-Gonzalez, R. R., Goforth, M. H., Morel, C. R., Subramanian, V., Mukundan, L., et al. (2007). Macrophage-specific PPAR $\gamma$ controls alternative activation and improves insulin resistance. Nature 447, 1116-1120. doi: 10.1038/nature05894

Oeckinghaus, A., Hayden, M. S., and Ghosh, S. (2011). Crosstalk in NF-кB signaling pathways. Nat. Immunol. 12, 695-708. doi: 10.1038/ni.2065

O'Neill, L. A. J., and Hardie, D. G. (2013). Metabolism of inflammation limited by AMPK and pseudo-starvation. Nature 493, 346-355. doi: 10.1038/nature11862

Palaga, T., Buranaruk, C., Rengpipat, S., Fauq, A. H., Golde, T. E., Kaufmann, S. H., et al. (2008). Notch signaling is activated by TLR stimulation and regulates macrophage functions. Eur. J. Immunol. 38, 174-183. doi: 10.1002/ eji.200636999

Rajamannan, N. M., Evans, F. J., Aikawa, E., Grande-Allen, K. J., Demer, L. L., Heistad, D. D., et al. (2011). Calcific aortic valve disease: not simply a degenerative process a review and agenda for research from the national heart and lung and blood institute aortic stenosis working group. Circulation 124, 1783-1791. doi: 10.1161/circulationaha.110.006767

Rajamannan, N. M., Subramaniam, M., Rickard, D., Stock, S. R., Donovan, J., Springett, M., et al. (2003). Human aortic valve calcification is associated with an osteoblast phenotype. Circulation 107, 2181-2184. doi: 10.1161/01.cir. 0000070591.21548 .69

Satoh, T., Takeuchi, O., Vandenbon, A., Yasuda, K., Tanaka, Y., Kumagai, Y., et al. (2010). The Jmjd3-Irf4 axis regulates M2 macrophage polarization and host responses against helminth infection. Nat. Immunol. 11, 936-944. doi: 10.1038/ ni. 1920

Sica, A., and Mantovani, A. (2012). Macrophage plasticity and polarization: in vivo veritas. J. Clin. Invest. 122, 787-795. doi: 10.1172/JCI59643

Song, R., Zeng, Q., Ao, L., Yu, J. A., Cleveland, J. C., Zhao, K. S., et al. (2012). Biglycan induces the expression of osteogenic factors in human aortic valve interstitial cells via Toll-like receptor-2. Arterioscler. Thromb. Vasc. Biol. 32, 2711-2720. doi: 10.1161/ATVBAHA.112.300116

Tedesco, S., Bolego, C., Toniolo, A., Nassi, A., Fadini, G. P., Locati, M., et al. (2015). Phenotypic activation and pharmacological outcomes of spontaneously differentiated human monocyte-derived macrophages. Immunobiology 220, 545-554. doi: 10.1016/j.imbio.2014.12.008

Wang, Q., He, Z., Huang, M., Liu, T., Wang, Y., Xu, H., et al. (2018). Vascular niche IL-6 induces alternative macrophage activation in glioblastoma through HIF-2alpha. Nat. Commun. 9:559. doi: 10.1038/s41467-018-03050-0

Wang, Y., He, F., Feng, F., Liu, X., Dong, G., Qin, H., et al. (2010). Notch signaling determines the M1 versus M2 polarization of macrophages in antitumor immune responses. Cancer Res. 70, 4840-4849. doi: 10.1158/0008-5472.CAN10-0269

Wang, Y. C., He, F., Feng, F., Liu, X. W., Dong, G. Y., Qin, H. Y., et al. (2010). Notch signaling determines the $\mathrm{m} 1$ versus $\mathrm{m} 2$ polarization of macrophages in 
antitumor immune responses. Cancer Res. 70, 4840-4849. doi: 10.1158/00085472.CAN-10-0269

Zeng, Q., Jin, C., Ao, L., Cleveland, J. J., Song, R., Xu, D., et al. (2012). Crosstalk between the Toll-like receptor 4 and Notch1 pathways augments the inflammatory response in the interstitial cells of stenotic human aortic valves. Circulation 126, S222-S230.

Zeng, Q., Song, R., Ao, L., Weyant, M. J., Lee, J., Xu, D., et al. (2013). Notch1 promotes the pro-osteogenic response of human aortic valve interstitial cells via modulation of ERK1/2 and nuclear factor- $\mathrm{b}$ activation. Arterioscler. Thromb. Vasc. Biol. 33, 1580-1590. doi: 10.1161/ATVBAHA.112.30 0912

Zeng, Q., Song, R., Fullerton, D. A., Ao, L., Zhai, Y., Li, S., et al. (2017). Interleukin-37 suppresses the osteogenic responses of human aortic valve interstitial cells in vitro and alleviates valve lesions in mice. Proc. Natl. Acad. Sci. U.S.A. 114, 1631-1636. doi: 10.1073/pnas.161966 7114

Zhang, W., Liu, H., Liu, W., Liu, Y., and Xu, J. (2015). Polycomb-mediated loss of microRNA let-7c determines inflammatory macrophage polarization via PAK1-dependent NF-kappa B pathway. Cell Death Differ. 22, 287-297. doi: $10.1038 /$ cdd. 2014.142
Zhou, D., Huang, C., Lin, Z., Zhan, S., Kong, L., Fang, C., et al. (2014). Macrophage polarization and function with emphasis on the evolving roles of coordinated regulation of cellular signaling pathways. Cell. Signal. 26, 192-197. doi: 10.1016/ j.cellsig.2013.11.004

Zhou, Y., Zhang, T., Wang, X., Wei, X., Chen, Y., Guo, L., et al. (2015). Curcumin modulates macrophage polarization through the inhibition of the toll-like receptor 4 expression and its signaling pathways. Cell Physiol. Biochem. 36, 631-641. doi: 10.1159/000430126

Conflict of Interest: The authors declare that the research was conducted in the absence of any commercial or financial relationships that could be construed as a potential conflict of interest.

Copyright (c) 2020 Zhou, Li, Su, Dong, Zong, Ma, Yang, Zuo, Zheng, Meng, Xu and Zeng. This is an open-access article distributed under the terms of the Creative Commons Attribution License (CC BY). The use, distribution or reproduction in other forums is permitted, provided the original author(s) and the copyright owner(s) are credited and that the original publication in this journal is cited, in accordance with accepted academic practice. No use, distribution or reproduction is permitted which does not comply with these terms. 